\title{
The majority of nurses first experience death in or before their first year of practice, and the experience can provoke feelings of helplessness, guilt and ongoing distress
}

\section{John Costello}

10.1136/eb-2012-101157

School of Nursing, Midwifery and Social Work, University of Manchester, Manchester, UK

\section{Correspondence to:}

John Costello

School of Nursing, Midwifery and Social Work, University of Manchester, University Place Oxford Road, Manchester M13 9PL, UK;

John.Costello@manchester.ac.uk

Commentary on: Kent B, Anderson NE, Owens RG. Nurses' early experiences with patient death: the results of an on-line survey of Registered Nurses in New Zealand. Int J Nurs Stud 2012;49:1255-65.

\section{Implications for practice and research}

- The study draws attention to the issue of patient death and its impact on staff well-being, moral and mental health, calling for these areas to be taken more seriously.

- The findings highlight a need for undergraduate death education to focus on end-of-life care.

- The study has implications for considering the positive impact death experiences can have on nurses.

- There is a need to review resources for managing patient death and to evaluate the impact a patient's death can have on nurses providing end-of-life care, particularly for student nurses and newly qualified staff.

\section{Context}

This study focuses on the death of patients in acute care contexts, an area identified as a source of anxiety for nurses with the potential to cause significant distress. ${ }^{12}$ Despite studies focusing on the impact of patient death, little is known of the specific stress experiences of nurses in their formative years.

\section{Methods}

The authors used online email invitations, requesting participants $(n=174)$ to complete a questionnaire exploring early clinical experiences of patient death. This number represented a 35\% response rate from registered nurses over 21; however, many of the first death experiences were experienced during their time as student nurses when they would have been under the age of 21 . The self-reported descriptive data pertained to the impact of the first death experience and what preparation and support structures were used.

\section{Findings}

The majority of patient death experiences (61\%) occurred when nurses were undergraduate students or in their first year of qualified practice (23\%). In particular, these experiences occurred while providing comfort care or conducting postmortem procedures with older patients. Nurses revealed that there were limited resources for managing early patient death experiences and a lack of preparation and support for coping with patient death. However, there were also positive aspects associated with their experiences, including feelings of making a difference, feeling privileged and being part of a team. The latter is one of the most significant experiences student nurses report about clinical placements. The majority of participants did not report explicitly upsetting experiences.

\section{Commentary}

The use of online surveys for data collection is becoming more common, although the topic of patient death experiences and their impact on staff is less common. One of the noteworthy aspects of the findings is that by monitoring such experiences, health service managers and/or nurse educators can learn a lot about how to prevent negative experiences, as well as highlighting the positive outcomes associated with such experiences.

The authors accept the limitations of using a convenience sample and self-reported data. They also express surprise at nurses' ability to recall such events so vividly. However, given that patient death, as reported, had such a significant impact, it is hardly surprising that these events are recalled with clarity, especially by nurses early in their careers.

The results largely confirm findings from other studies which indicate that nurses are often significantly affected by patient death. ${ }^{1-3}$ The authors point out that participants received education about patient death, although the amount was not specified and it did not seem to prepare them. It should be noted that, internationally, not all nursing curricula include death education as part of undergraduate programmes. Future research could explore whether education can better prepare nurses for managing patient death positively. The majority of reported experiences were perceived as significant, vividly remembered, but not necessarily distressing. This supports the view highlighted by others that, such experiences have potential for personal growth arising from caring for patients before and after death. ${ }^{3}$

The authors point to a need for further discussion and research that can be generalisable outside New Zealand. They also call for caution about the potential of the newly developed 'Patient Death Impact Scale'. The sensitivity of the tool is limited, and a high score could just as well indicate a positive or a negative overall experience. A much more sophisticated humanistic approach would be to have a verbal debrief with a competent senior practitioner who is able to identify and acknowledge the value and importance of such experiences, and offer appropriate emotional support and professional guidance where required.

Competing interests None. 


\section{References}

1. Beck C. Secondary traumatic stress in nurses: a systematic review. Arch Psychiatr Nurs 2011;25:1-10.
2. Copp G. Facing impending death. London, UK: Nursing Times books, 1999.

3. Wilson J, Kirshbaum M. Effects of patient death on nursing staff: a literature review. Br J Nurs 2011;20:559-63. 